\title{
ALPK1 Expression Is Associated with Lymph Node Metastasis and Tumor Growth in Oral Squamous Cell Carcinoma Patients
}

\author{
Po-Ku Chen, ${ }^{* \dagger+}$ Chun-Hung Hua, ${ }^{\S}$ Hui-Ting Hsu,, Tzer-Min Kuo, ${ }^{*}$ Chia-Min Chung, ${ }^{* \|}$ Chi-Pin Lee, ${ }^{*}$ Ming-Hsui Tsai, \\ Kun-Tu Yeh, ** and Ying-Chin Ko*
}

From the Environment-Omics-Diseases Research Centre, ${ }^{*}$ the Rheumatology and Immunology Center, ${ }^{\dagger}$ the Translational Laboratory, ${ }^{\ddagger}$ Rheumatic Disease Research Center, and the Department of Otorhinolaryngology, ${ }^{\S}$ China Medical University Hospital, and the Graduate Institute of Biomedical Sciences, ${ }^{\|}$China Medical University, Taichung; and the Departments of Surgical Pathology and Pathology, ** Changhua Christian Hospital, Changhua, Taiwan

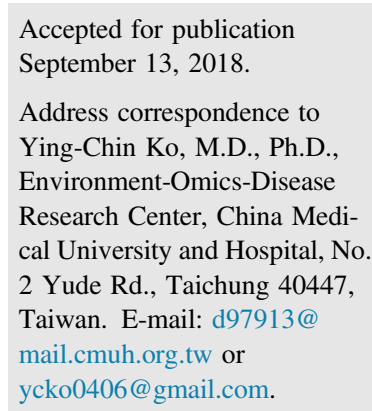

\begin{abstract}
Oral squamous cell carcinoma (OSCC) is the most common malignant cancer, with high mortality rates in advanced stages. Recent studies have shown that the expression of ALPK1 mRNA and its inhibitory differentiation function are associated with cancer progression. However, the expression and clinicopathologic features of ALPK1 in OSCC remain unexplored. Herein, the authors investigated the expression patterns of ALPK1 in 39 matched OSCC patients and examined the relationship between ALPK1 protein expression and clinicopathologic factors using immunohistochemical scores. Using Western blot analysis, ALPK1 expression was found to be significantly higher in tumor tissues than that in nontumor tissues. Through an immunoreactive scoring system, a significantly higher number of advanced-stage tumor size T4 and lymph node metastasis N2 exhibited higher ALPK1 expression levels than that exhibited by T1/T2/T3 tumors and N0/N1. In addition, ALPK1 protein expression was aberrant in malignant oral cancer cell lines compared with that in pre-malignant oral epithelial cells, whereas minimal expression was observed in normal oral epithelial cells. Knockdown of ALPK1 resulted in a significant reduction in cell growth, migration, and invasion capacity in vitro. Consequently, expression of $\mathrm{N}$-cadherin and vimentin decreased in ALPK1-deficient cells. Thus, these results suggest that ALPK1 serves as a potential biomarker and target for OSCC development in late stages. (Am J Pathol 2019, 189: 190-199; https://doi.org/10.1016/j.ajpath.2018.09.003)
\end{abstract}

Oral cancer is the sixth most common malignant cancer in the world and accounts for approximately $5 \%$ of malignant tumors in developing countries. More than 500,000 new cases of oral cancer are diagnosed each year. ${ }^{1}$ Studies on the clinical pathology of oral cancer have shown that late-stage oral cancer (T3/T4) has a significantly higher recurrence rate than an early stage (T1/T2) and is related to incidence of secondary metastasis. $^{2,3}$ Unfortunately, oral squamous cell carcinoma (OSCC) is usually not detected until advanced stages, which may help to explain the high mortality rate and poor overall survival. ${ }^{4}$ Although a number of neutralization antibodies and tyrosine kinase inhibitors for the epidermal growth factor receptor (EGFR) signaling pathway have been developed, the only approved treatment for advance stage OSCC is the anti-EGFR monoclonal antibody cetuximab. ${ }^{6}$ Despite substantial progress in therapeutic approach and management, the mortality rate of OSCC has not changed. Therefore, it is necessary to develop a new therapeutic strategy to reduce the mortality of advanced tumors.

Alpha-kinase 1 (ALPK1) belongs to a newly discovered protein kinase family that has no sequence homology to the conventional protein kinases, and its catalytic domains appear to be homologous with the catalytic domain of myosin heavy chain kinase A from Dictyostelium. ${ }^{7-9}$ ALPK1 functions as a

Supported by Ministry of Science and Technology grants MOST1062314-B-039-016-MY3 and MOST106-2314-B-039-017-MY3 (Y.-C.K.), and China Medical University Hospital grants DMR-107-108 and DMR108-117 (Y.-C.K.)

Disclosures: None declared. 
carrier vesicle for apical protein transporter in epithelium cells through phosphorylating myosin $1 \mathrm{~A} .{ }^{10}$ In inflammatory cells, ALPK1 also participates in inflammatory responses that induce expression of proinflammatory cytokines, such as tumor necrosis factor alpha (TNF- $\alpha$ ), IL-1 $1 \beta$, and IL-8, through the activation of nuclear factor- $\mathrm{kb}$ and mitogen-activated protein kinase. ${ }^{11-13}$ Through proteomics and biochemical analysis, ALPK1 could directly phosphorylate myosin IIA to modulate TNF- $\alpha$ secretion under monosodium urate treatment. ${ }^{14}$ Results of a genome-wide association study suggest that ALPK1 contributes to chronic inflammation of the kidneys in diabetic glomerulosclerosis. ${ }^{15}$ ALPK1 has also been shown to play a role in the immune response, acting as a critical mediator for interacting between Helicobacter pylori, gram-negative bacteria, and cell-cell contact. ${ }^{16,17}$ A recent study provided evidence that APLK1 plays an inhibitory role in the differentiation of breast cancer, ${ }^{18}$ and $A L P K 1$ mRNA expression is correlated with lung and colorectal cancer progression, ${ }^{19}$ However, the association of ALPK1 expression with clinicopathologic features and the cancer progression of OSCC remains unclear.

Here, the clinicopathologic features of expression patterns of ALPK1 in OSCC were investigated, and its potential correlations with TMN classification were examined. ALPK1 was found to be highly expressed in OSCC and moderate dysplasia cells in vitro, whereas ALPK1 was only minimally expressed in normal oral keratinocytes. Moreover, evidence was found that ALPK1 is required for cell migration, invasion, and proliferation in OSCC cells. Additionally, the effect of ALPK1 inhibition on the levels of proteins related to the epithelium-to-mesenchymal transition (EMT) was also determined. Taken together, the results suggest that ALPK1 functions as a tumor progression factor and serves as a diagnostic biomarker and therapeutic target for OSCC.

\section{Materials and Methods}

\section{Patients and Tissues}

The protocol of this study was approved by the ethics committee of China Medical University Hospital. The clinical study was conducted according to the regulations of China Medical University Hospital, Taiwan. Primary OSCC specimens (tumor) and matching adjacent normal surrounding mucosa specimens (nontumor) were obtained from surgical samples of 39 patients who were treated at the China Medical University Hospital. All patients were $>18$ years of age, met eligibility criteria for this study, and were informed of the detailed study protocol, required to sign consent forms, and instructed to complete a standardized questionnaire. The location of tumor tissues was recorded and classified according to the International Union against Cancer TNM classification system (8th edition). ${ }^{20}$

\section{OSCC Cell Lines and Transfection}

Human oral keratinocyte (HOK) and dysplastic oral keratinocyte (DOK) cells were purchased from the European Collection of Animal Cell Cultures and maintained in oral keratinocyte medium (Sciencell, Carlsbad, CA) as described in the manual. SCC-9, SAS (derived from a primary tongue SCC), and HSC-3 (derived from a lymph node metastasis of tongue SCC) cells were purchased from the Bioresource Collection and Research Center, Taiwan, and maintained in DMEM/F12 (Invitrogen, Carlsbad, CA) medium supplemented with $10 \%$ fetal bovine serum and $5 \mu \mathrm{g} / \mathrm{mL}$ hydrocortisone. OC 3 cells (primary cultured OSCC derived from tongue cancer patients) were kindly provided by Dr. ShuChun Lin (National Yang Ming University, Taipei, Taiwan)
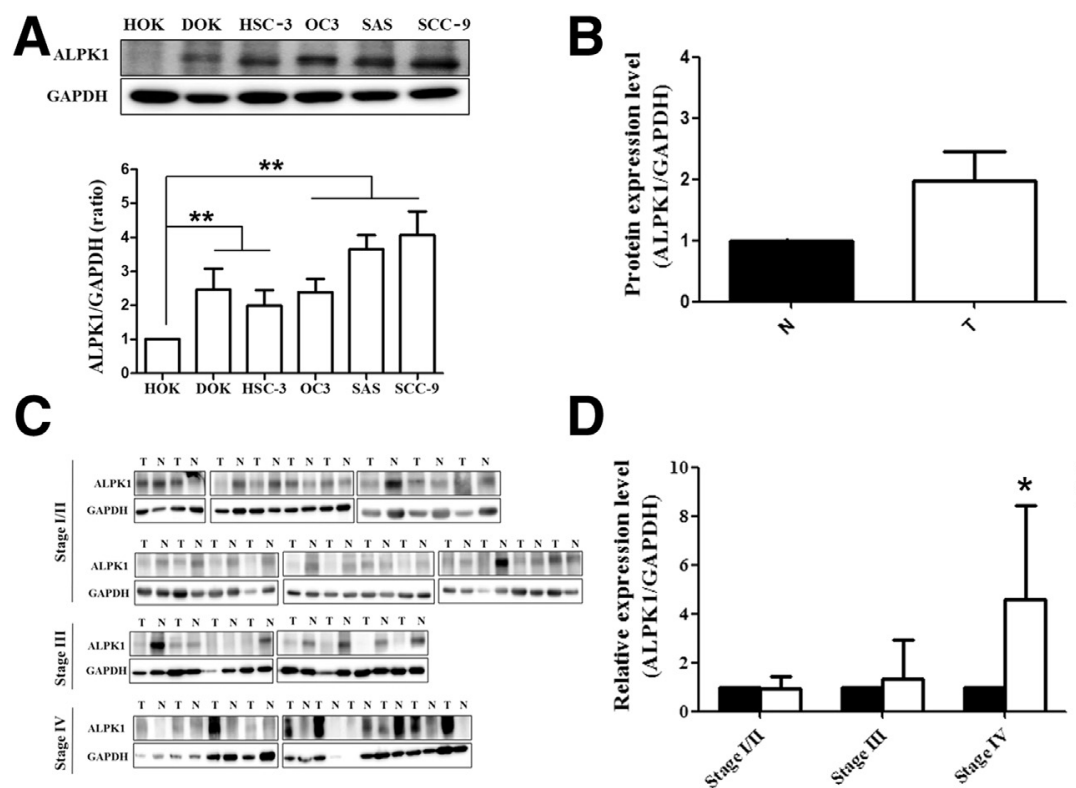

D

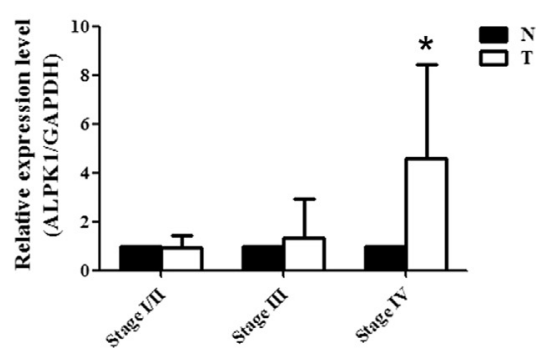

Figure 1 Expression of ALPK1 in oral squamous cell carcinoma (OSCC) cell lines and 39 OSCC specimens and matched nontumor specimens. A: Western blot analysis of ALPK1 expression in human oral keratinocyte (HOK), dysplasia oral keratinocyte (DOK), and oral cancer cells lines. B: Surgical biopsy of tumor samples and matched nontumor samples collected from patients with oral cancer of different stages. Samples were analyzed for ALPK1 expression by Western blot analysis. GAPDH and $\alpha$-tubulin were used as loading controls. C: Ratio of ALPK1 expression in normal nontumor and tumor samples obtained from patients. Band intensity was quantified by ImageJ software version 1.52a (NIH, Bethesda, MD; $h t t p: / / i m a g e j . n i h . g o v / i j)$. D: ALPK1 expression in OSCC patients of four different stages was analyzed by ImageJ software, with normalization of ALPK1 against GAPDH. Data were analyzed using paired $t$-tests. Data are expressed as means \pm SD. $n=39$ (B, patients). ${ }^{*} P<0.05$, ${ }^{* *} P<0.01$. N, normal nontumor tissue; $\mathrm{T}$, tumor tissue. 
and cultured in a 1:2 mixture of DMEM-F12 and Keratinocyte-SFM (Invitrogen, Carlsbad, CA) with $10 \%$ fetal bovine serum. All cells were cultured in a humidified $5 \% \mathrm{CO}_{2}$ atmosphere at $37^{\circ} \mathrm{C}$. To examine the biological functions of ALPK1 expression in OSCC, cells were transiently transfected with control or human ALPK1 siRNAs that were purchased from Dharmacon (ALPK1 siRNAs: catalog number M-005009-02-0020; control siRNAs: catalog number D-001810-10-05; Dharmacon, Lafayette, CO) and Invitrogen by using the RNAiMAX transfection reagents (Invitrogen). After 24 to 48 hours transfection, cells were harvested for subsequent detection of the level of ALPK1 expression by Western blotting.

\section{Immunohistochemical Staining and Evaluation}

For the whole process of immunohistochemical staining, the paraffin-embedded tissue ( $4 \mu \mathrm{m}$ thickness) were used as described previously. ${ }^{21}$ Briefly, nontumor or tumor tissue sections were deparaffinized, rehydrated, and then stained with hematoxylin and eosin for histological evaluation. For evaluation of ALPK1 expression, the ALPK1-specific antibody (Aviva Systems Biology, San Diego, CA) was used for immunohistochemical detection. The immunohistochemical images have been evaluated and scored by two professional pathologists (H.-T.H. and K.-T.Y.). Each tumor was given a score according to the intensity of the nuclear and cytoplasmic staining: no staining, 0 ; weak staining, $1+$; moderate staining, $2+$; and strong staining, $3+$.On the basis of the ratio of staining, ALPK1 values in cancer cells of tumor tissue were divided into 4 levels: 1 , staining values $0 \%$ to $30 \% ; 2$, staining values $31 \%$ to $50 \%$; 3 , staining values $51 \%$ to $80 \%$; and 4 , staining values $>80 \%$. For the immunoreactive scoring system, cutoff scores $\leq 6$ and $>6$ were defined as low and high expression levels, respectively. ${ }^{22}$

\section{Migration and Invasion Assay}

Cell migration and invasion was performed in a 24-well Transwell chamber with a pore size of $8 \mu \mathrm{m}$ (Corning, Bedford, MA). For invasion assay, the membrane was precoated with $100 \mu \mathrm{L}$ of Matrigel (dilution 1:2; Corning) for 1 hour. Cells transfected with control (siCon) or ALPK1 siRNA (siALPK1) $\left(5 \times 10^{4}\right)$ were seeded onto the upper chamber with Matrigel (invasion assay) or without Matrigel (migration assay) and incubated in serum-free medium for the indicated time periods. The lower chamber was filled with $10 \%$ fetal bovine serum medium. The membranes were stained using LiuA/LiuB staining reagent for $2 \mathrm{mi}$ nutes. Nonmigrated cells on the upper side of the membrane were wiped off with a cotton swab. The numbers of migrated or invaded cells were counted in four randomly
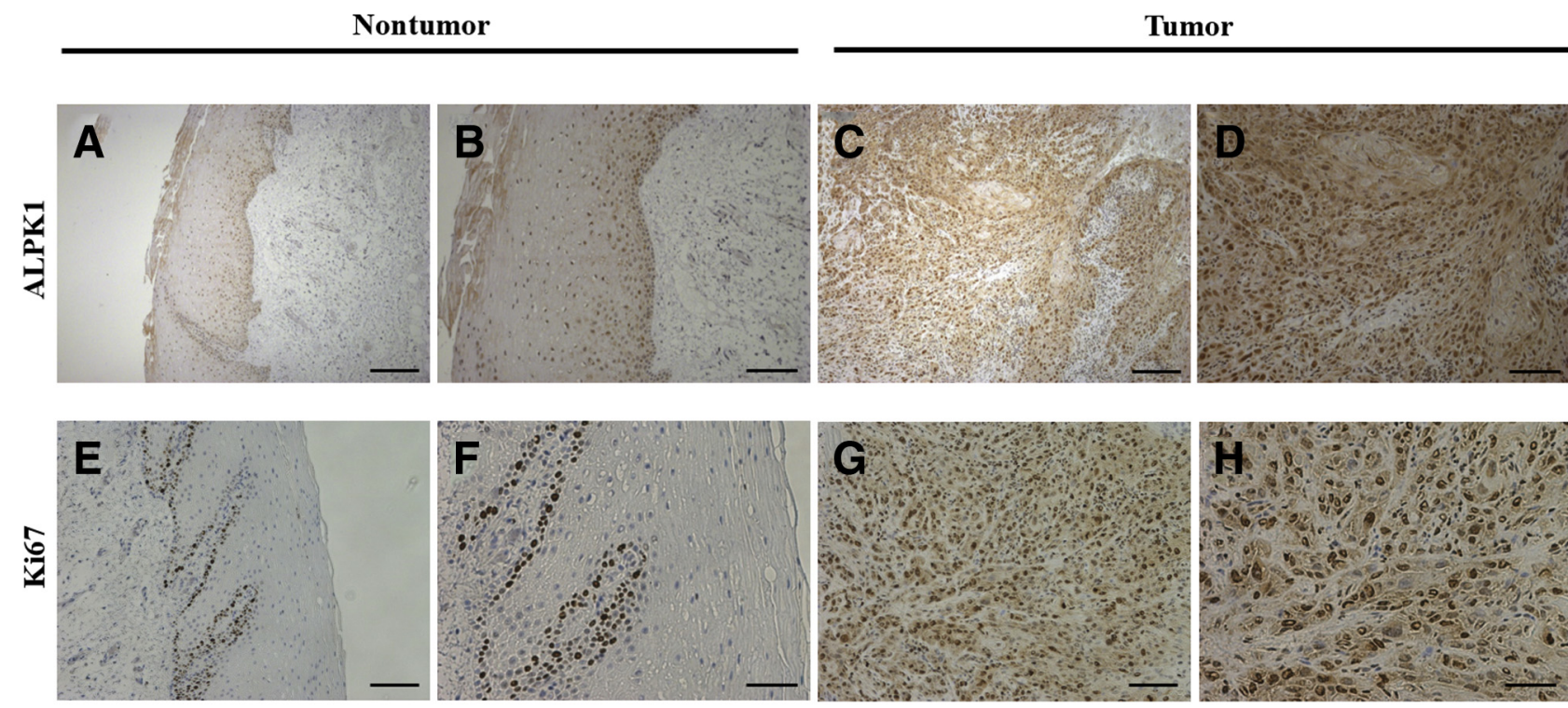

Kidney
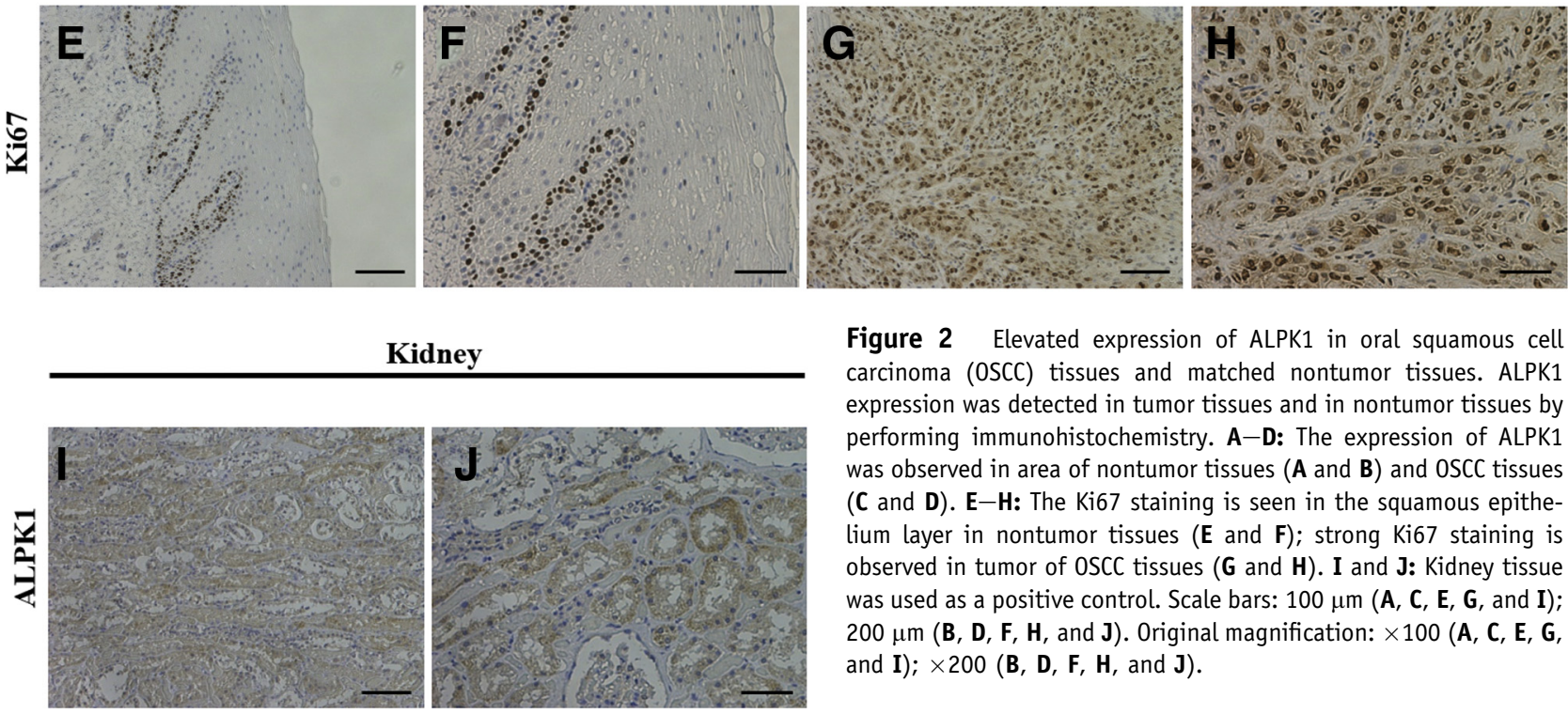

Figure 2 Elevated expression of ALPK1 in oral squamous cell carcinoma (OSCC) tissues and matched nontumor tissues. ALPK1 expression was detected in tumor tissues and in nontumor tissues by performing immunohistochemistry. A-D: The expression of ALPK1 was observed in area of nontumor tissues (A and $\mathbf{B}$ ) and OSCC tissues ( $\mathbf{C}$ and $\mathbf{D})$. $\mathbf{E}-\mathbf{H}$ : The Ki67 staining is seen in the squamous epithelium layer in nontumor tissues ( $\mathbf{E}$ and $\mathbf{F}$ ); strong Ki67 staining is observed in tumor of OSCC tissues ( $\mathbf{G}$ and $\mathbf{H}$ ). I and $\mathbf{J}$ : Kidney tissue was used as a positive control. Scale bars: $100 \mu \mathrm{m}(\mathbf{A}, \mathbf{C}, \mathbf{E}, \mathbf{G}$, and I); $200 \mu \mathrm{m}(\mathbf{B}, \mathbf{D}, \mathbf{F}, \mathbf{H}$, and J). Original magnification: $\times 100(\mathbf{A}, \mathbf{C}, \mathbf{E}, \mathbf{G}$, and $\mathbf{I}) ; \times 200(\mathbf{B}, \mathbf{D}, \mathbf{F}, \mathbf{H}$, and $\mathbf{J})$. 
Table 1 Demographic and Characteristics among Oral Cancer Patients

\begin{tabular}{lrr}
\hline Factor & $N$ & $\%$ \\
\hline Sex & & \\
$\quad$ Male & 35 & 89.74 \\
$\quad$ Female & 4 & 10.26 \\
Age, years & & \\
$\quad \leq 50$ & 17 & 43.59 \\
$>50$ & 19 & 48.72 \\
Unknown & 3 & 7.69 \\
Tumor size & & \\
I & 9 & 23.08 \\
II & 12 & 30.77 \\
III & 8 & 20.51 \\
IV & 10 & 25.64 \\
Lymph node & & \\
N0 & 8 & 20.51 \\
N1 & 13 & 33.33 \\
N2 & 15 & 38.46 \\
N3 & 1 & 2.56 \\
Unknown & 2 & 5.13 \\
Metastasis & & \\
No & 39 & 100.00 \\
Yes & 0 & 0.00 \\
\hline
\end{tabular}

selected high-power fields $(\times 10$ objective lens $)$ using a Canon digital camera (Tokyo, Japan) with a microscope.

\section{Proliferation Assay}

To evaluate the effect of ALPK1 on cell proliferation ability, SAS and HSC-3 cells transfected with siCon or siALPK1 for 24 hours were plated onto 96-well plates $\left(2.5 \times 10^{3}\right.$ cells per well $)$ and incubated for 24 or 48 hours. The cell growth rate was assessed using the 3-(4,5dimethylthiazol-2-yl)-2,5-diphenyltetrazolium bromide (MTT) assay as described previously. ${ }^{19}$ The absorbance was measured in a microplate reader at $570 \mathrm{~nm}$.

\section{Cytokines Array}

The Human Cytokine Protein Array (Bio-Rad Laboratories, Hercules, CA) was used for cytokine profiling of OSCCs.
Conditioned medium was collected from HSC-3, SAS, and OC3 cells with transfection of siALPK1 or siCon for 48 hours. Cytokine profiles of each group were then generated using the Bio-Plex Multiplex assay (Bio-Rad Laboratories) according to the manufacturer's instructions.

\section{Western Blot Analysis}

The whole cells were lysed by radioimmunoprecipitation assay buffer (Thermo Fisher Scientific, Rockford, IL) containing a cocktail of protease inhibitors (Roche Diagnostics, Indianapolis, IN) for 20 minutes on ice and then centrifuged at 15,800 $\times g$ for 20 minutes at $4^{\circ} \mathrm{C}$. The samples were then separated by SDS-PAGE and electrotransferred to polyvinylidene fluoride membranes (Millipore, Billerica, MA). Western blotting was performed with primary antibodies against ALPK1, E-cadherin, N-cadherin, and vimentin (Cell Signaling Technology, Danvers, MA). GAPDH (Millipore) and $\alpha$-tubulin (GeneTex, Hsinchu City, Taiwan) were used as loading controls.

\section{Statistical Analysis}

The $\chi^{2}$ square test and McNemar test were used to examine the difference in tumor location and adverse oral habits between grouped OSCC participants. The paired data were then analyzed in $2 \times 2$ tables by using the McNemar test. For statistical purposes, no change and/or equivalent expression from nontumor to tumor tissue were defined as unchanged, and change expression, as high expression. This two-point scale has been used in several other studies for analyzing ALPK1 expression in OSCC. For cell culture experiments, the statistical differences were evaluated using the $t$-test accordingly.

\section{Results}

The Expression of ALPK1 in OSCC Cell Lines and 39 Paired OSCC Specimens

To investigate the potential association between ALPK1 protein expression and oral cancer progression, HOK, DOK, and four OSCC cell lines (HSC-3, OC-3, SAS, and SCC-9)

Table 2 Clinicopathologic Factors Associated with ALPK1 Expression in Paired Nontumor and OSCC Patients by Immunoreactive Scoring Systems

\begin{tabular}{|c|c|c|c|c|c|c|c|c|c|}
\hline \multirow[b]{2}{*}{ ALPK1 expression } & \multirow[b]{2}{*}{$N$} & \multicolumn{3}{|l|}{ Nontumor } & \multirow[b]{2}{*}{$N$} & \multicolumn{3}{|l|}{ Tumor } & \multirow{2}{*}{$\begin{array}{l}P \text { value (nontumor } \\
\text { versus tumor) }\end{array}$} \\
\hline & & $\operatorname{Low}(n, \%)$ & $\operatorname{High}(n, \%)$ & $P$ value & & $\operatorname{Low}(n, \%)$ & $\operatorname{High}(n, \%)$ & $P$ value & \\
\hline \multicolumn{10}{|l|}{ Tumor size } \\
\hline $\mathrm{T} 3$ & 7 & $5(71.48)$ & $2(28.52)$ & & 7 & $2(28.52)$ & $5(71.48)$ & & $>0.05^{*}$ \\
\hline T4 & 9 & $9(100)$ & $0(0)$ & & 10 & $1(11.1)$ & $8(89.9)$ & & $<0.0001^{\dagger}$ \\
\hline \multicolumn{10}{|l|}{ Lymph node } \\
\hline
\end{tabular}

*Calculated by $\chi^{2}$ test.

${ }^{\dagger}$ Calculated by Fisher exact test. 
Table 3 The Alternation of ALPK1 Expression in Pairs of Noncancer and OSCC Tissue with Tumor Size and Lymph Node Stage

\begin{tabular}{lrlll}
\hline ALPK1 expression & $N$ & Positive, $n(\%)$ & Negative, $n(\%)$ & $P$ value \\
\hline Tumor size & & & & \\
$\quad$ I-III & 26 & $14(53.84)$ & $12(46.16)$ & $<0.001$ \\
$\quad$ IV & 9 & $9(100)$ & $0(0)$ & \\
$\quad$ Total & 35 & $23(65.71)$ & $12(34.29)$ & \\
Lymph node & & & & \\
$\quad$ N0/N1 & 20 & $12(60)$ & $8(40)$ & $<0.05$ \\
N2 & 14 & $11(78.57)$ & $3(21.43)$ & \\
$\quad$ Total & 34 & $23(67.64)$ & $11(33.36)$ & \\
\hline
\end{tabular}

Calculated by McNemar test.

were examined via Western blot analysis. Compared with that in HOK, ALPK1 expression was higher in DOK, HSC3 , and OC-3 cells; it was higher in SAS and SCC-9 compared with that in DOK, HSC, and OC-3 cells (Figure 1A). Given evidence of high expression of ALPK1 in aggressive forms of OSCC, the role of ALPK1 was explored in predicting the clinical outcome of OSCC. In particular, the expression of ALPK1 protein was evaluated in 39 tissue samples of oral cancer and matched noncancerous tissues. Higher levels of ALPK1 protein were found in tumor tissues compared with nontumor tissues (1.91-fold; $P=0.055$ ) (Figure 1B). Further, the expression levels of ALPK1 protein were associated with tumor size T4 $(P=0.033)$, but not T1/T2 $(P=0.492)$ or T3 $(P=0.677)$ (Figure 1, C and D). These results indicate that ALPK1 is highly correlated to the progression of OSCC.

\section{Relationship between ALPK1 and Clinicopathologic Factors}

To study the expression of ALPK1 pattern in patients with OSCC, immunohistochemistry was performed on tumor tissue and matched nontumor tissue. ALPK1 was highly expressed in OSCC, whereas minimal expression was detected in nontumor tissue (Figure 2, A and B). The distribution of ALPK1 in OSCC tissue was located both in the nucleus and cytoplasm (Figure 2, C and D). The staining of Ki67 in nontumor tissue was observed in nuclei in the squamous keratinocyte layer (Figure 2, E and F); whereas strong expression was detected in OSCC tissue (Figure 2, G and $\mathrm{H}$ ). The staining of ALPK1 in kidney tissue served as a positive control (Figure 2, I and J).

The demographic and clinicopathologic characteristics for OSCC patients are shown in Table 1. A predominant proportion of oral malignant cases were male (95\%), as compared with female $(5 \%)$. Among the cancer patients, $46.15 \%$ and $41.12 \%$ were found to have tumors with stage III/IV and lymph node metastasis of N2/N3, respectively. The association between clinicopathologic factors and ALPK1 expression via immunohistochemical scoring (calculated by the immunoreactive scoring system) is shown in Tables 2 and 3. Compared with nontumor tissue, higher levels of ALPK1 expression in nuclei of tumor tissue were found, not only in T1/T2 $(P=0.0248)$ and T4 $(P=0.001)$, but also in N0/N1 $(P=0.008)$ and N2 $(P=0.0016)$. However, no significant difference in T3 were found $(P=0.108)$ (Table 2). Furthermore, ALPK1 immunoreactivity in tumor tissue was found in different $\mathrm{T}$ stages $(P=0.028)$ (Table 2$)$. It was also examined whether ALPK1 expression levels in OSCC tissues changed from nontumor to cancerous OSCC tissues. A dramatic increase in ALPK1 levels in T4 (100\%, 9 of 9) and N2 (78.57\%, 11 of 14) was observed compared with T1/T2/T3 (53.84\%; $P=0.0001)$ in N0/N1 $(60 \%, 12$ of $20 ; P=0.0388)$ (Table 3). Taken together, these results show that a significantly higher number of advanced-stage $\mathrm{T} 4$ and $\mathrm{N} 2$ exhibit higher ALPK1 expression levels than that exhibited by T1/ T2/T3 tumors and N0/N1.

\section{Decreased ALPK1 Expression by siRNA Influences OSCC Cell Viability}

Given the observed clinically significant link between ALPK1 overexpression and $\mathrm{T} 4$ staging, different expression levels of ALPK1 were used in OSCC cells to evaluate the effect of ALPK1 on cell proliferation via an MTT assay. Knockdown of ALPK1 partially reduced cell viability of HSC-3 and OC-3 (Figure 3, A and C, Supplemental Figure S1), which is related to moderate expression levels of ALPK1 protein. However, knockdown of ALPK1 also significantly inhibited the
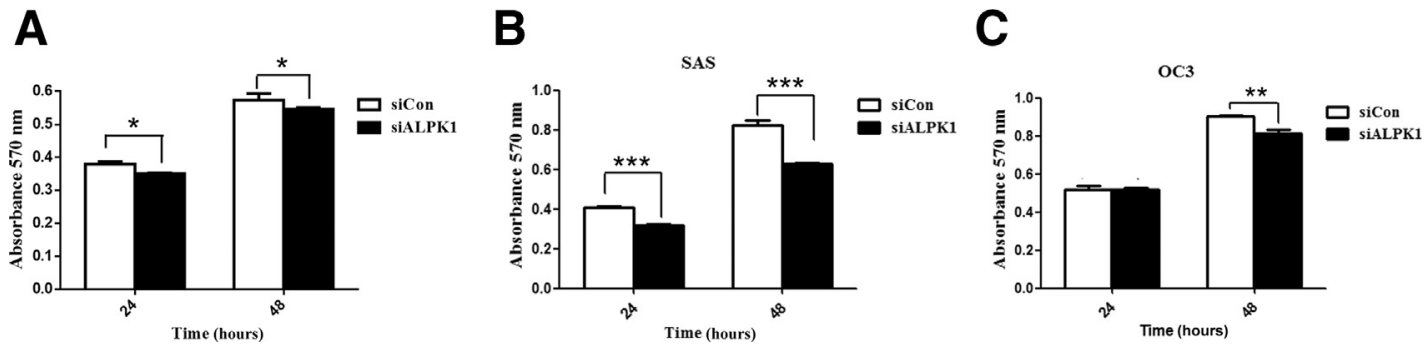

Figure 3 Down-regulation of ALPK1 expression by siRNA inhibits oral squamous cell carcinoma (OSCC) cell proliferation. HSC-3 (A), SAS (B), and OC3 (C) cell proliferation was measured by MTT assays at the indicated times after transient transfection with ALPK1-specific siRNA (siALPK1) and a nonspecific siRNA [negative control (siCon)]. Statistical significance was determined by $t$-test. Data are expressed as means \pm SD. $n \geq 3$ independent experiments. ${ }^{\star} P<0.05$, ${ }^{* *} P<0.01$, and ${ }^{* * *} P<0.001$ 
A

HSC-3 siCon SiALPK1

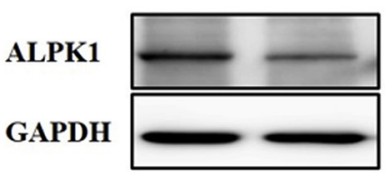

D
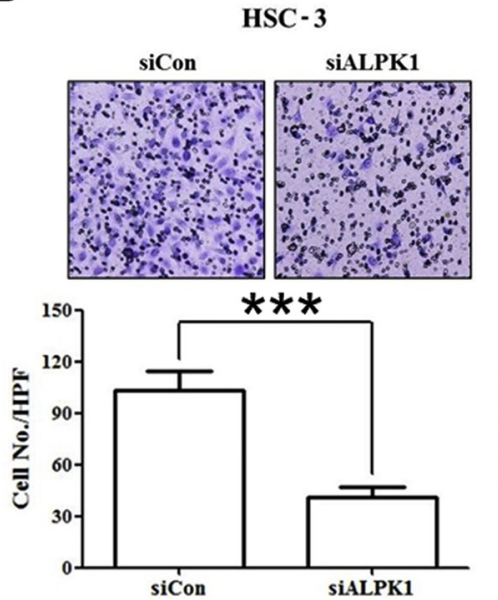

G
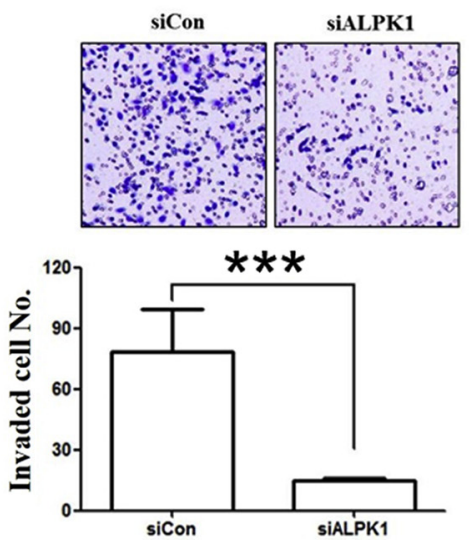

B

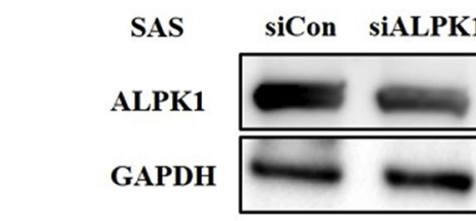

E
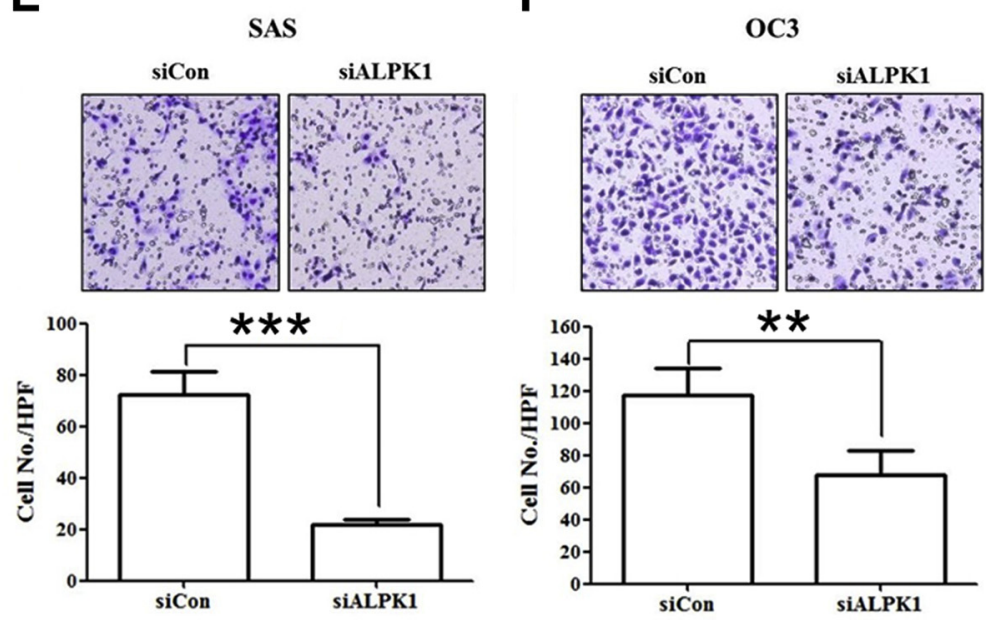

H
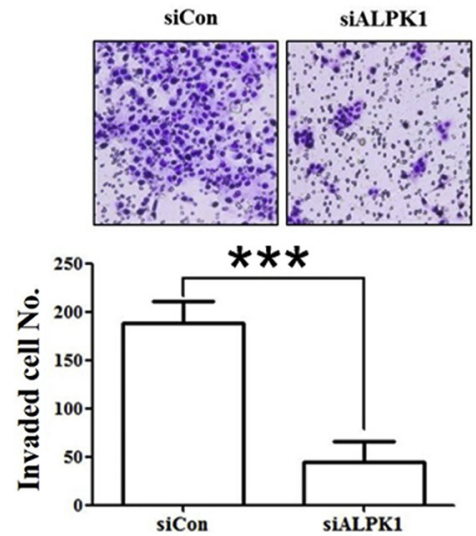

Figure 4 Knockdown of ALPK1 markedly reduces the cell migration and invasion ability of oral squamous cell carcinoma (OSCC). ALPK1 expression in HSC-3 (A), SAS (B), and OC3 (C) cells, which were transiently transfected with ALPK1-specific siRNA (siALPK1) and a nonspecific siRNA [negative control (siCon)], were analyzed at 24 hours. The migrated HSC-3 (D), SAS (E), OC3 (F) cells and the invaded HSC-3 (G) and SAS (H) cells were stained and then counted using a Canon digital camera with a microscope. Numbers of migrated cells represent the average counts. Statistical significance was determined by $t$-test. Data are presented as means \pm SD. $n \geq 3$ independent experiments; $n=5$ (D-H, fields of view). ${ }^{* *} P<0.01,{ }^{* \star} P<0.001$. Original magnification: $\times 10$ (D-H). HPF, high-powered field.

proliferation of SAS cells (Figure 3B, Supplemental Figure S1), which showed high expression of ALPK1 at 24 and 48 hours. The results suggest that ALPK1 is able to enhance cell proliferation in vitro.

\section{ALPK1 Promotes 0SCC Cell Migration and Invasion}

The clinical significance of ALPK1 with respect to lymph node metastasis was evaluated (Table 3 ). To test whether ALPK1 is involved in the biological functions of OSCC, the effect of ALPK1 depletion was examined with an assay that measures migration and invasion. First, all OSCC cells were transfected with siALPK1 for 24 hours, followed by reseeding the cells into the Transwell chamber. Knockdown efficiency of ALPK1 was examined in HSC-3, SAS, and OC-3 cells via Western blot analysis (Figure 4, A-C). Compared with the siCon group, ALPK1 inhibition significantly reduced cell migration ability (Figure 4, D-F). It was also tested whether ALPK1 plays an important role in cell invasion ability in a Transwell chamber with Matrigel. A dramatic decrease was observed in cell invasiveness of HSC-3 (Figure 4G) and SAS (Figure 4H) cells after ALPK1 depletion. These results suggest that ALPK1 participates in the development of tumor metastasis. 
A



B

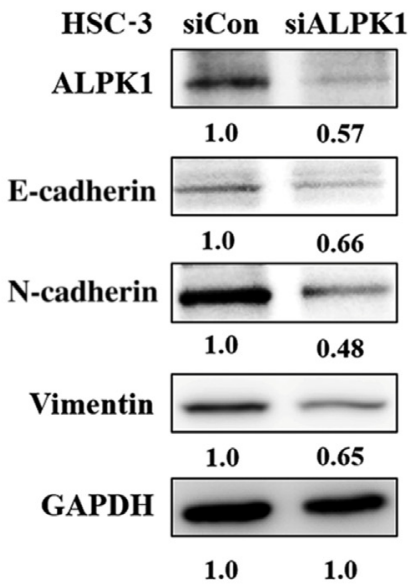

C

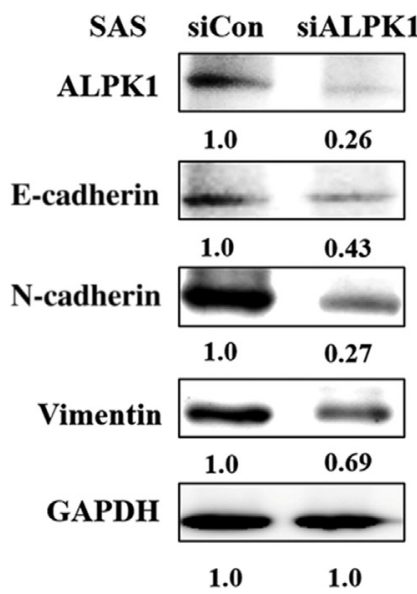

Figure 5 Knock down of endogenous ALPK1 in oral squamous cell carcinoma (OSCC) suppresses epithelium-to-mesenchymal transition (EMT)-related protein expression. A: Morphology of HSC-3 cells that were transiently transfected with ALPK1-specific siRNA (siALPK1) and a nonspecific siRNA [negative control (siCon)], is shown at 48 hours. B and C: Expression levels of E-cadherin, N-cadherin, and vimentin in HSC (B) and SAS (C) cells were analyzed by Western blot analysis using antibodies specific for E-cadherin, $\mathrm{N}$-cadherin, and vimentin.

\section{Knockdown of Endogenous ALPK1 in 0SCC Decreases EMT-Related Protein Expression}

Given that ALPK1 enhanced cell migration and invasion in OSCC, it was further investigated whether ALPK1 could affect the expression of several EMT markers in ALPK1 knockdown cells. The cell morphology between siCon and siALPK1 on HSC-3 did not change (Figure 5A). The mesenchymal markers $\mathrm{N}$-cadherin and vimentin were significantly decreased in the APLK1-depleted HSC-3 (Figure 5B) and SAS (Figure 5C) cells, but there was slight change in the epithelial marker, E-cadherin, compared with siCon cells. These results indicate that ALPK1 promotes cell mobility via regulation of the EMT pathway.

\section{Discussion}

To date, few studies have evaluated the role of ALPK1 in cancer development. Results of a recent clinical study suggest that mutation of ALPK1 results in the loss of biological function in lung and colorectal cancer patients and that ALPK1 mRNA expression correlates with tumorigenesis. ${ }^{19}$ In triple mutation breast cancer, ALPK1 cells can modulate tumorigenic ability in vivo and in vitro. ${ }^{18}$ However, there has been little evidence for an association between ALPK1 expression and clinicopathologic signatures in OSCC. Here, higher expression of ALPK was demonstrated in OSCC than in nontumor tissue. Moreover, these findings demonstrate a link between higher expression levels of ALPK1 and lymph node metastasis and advanced stage. In addition, it was observed that ALPK1 is required for cell migration, invasion, and proliferation in OSCC cells. Together, these results suggest that ALPK1 may be a new therapeutic target and diagnostic marker in late-stage OSCC.
EMT is essential for tumor progression and metastasis, and has been shown to predict poor survival and prognosis in cancer patients. ${ }^{23,24}$ When EMT process was driven by an inducer, the expression of epithelial markers was reduced and the expression of mesenchymal markers was induced. ${ }^{25}$ Recent studies have shown that $\mathrm{N}$-cadherin and vimentin show highly positive expression in malignant cells of OSCC patients. ${ }^{26-28}$ In line with these results, significant associations were found among ALPK1, N-cadherin, and vimentin expression in SAS and HSC3 cells (Figure 5). These results imply that ALPK1 increases cancer cell invasion through EMT regulation.

Previous reports show that overexpression or knockdown of ALPK1 in lung and colorectal cancer cells can modulate cell migration ability. ${ }^{19}$ Using a genetic manipulation approach in ALPK1 mutation mice, it was shown that ALPK1 may control fine motor activity. ${ }^{29}$ These results are consistent with those of the present study, in that deficient ALPK1 expression may inhibit cell migration and invasion (Figure 4). Myosin activation by phosphorylation of its heavy chain or light chain modulates actin filament reorganization, which subsequently promotes cell migration, invasion, and adhesion. ${ }^{30,31}$ However, it has been shown that myosin families have paradoxical effects on the regulation of cancer cell mobility. ${ }^{32-34}$ ALPK1 phosphorylates and activates myosin IA and myosin IIA to become a transporter in the lipid raft membrane and molecular trafficking. ${ }^{10,14}$ Some essential stimulators such as bacteria, testosterone, and monosodium urate monohydrate can activate ALPK1 to become a master regulator via interacting with downstream proteins in the inflammation process. TGF- $\beta$ is a regulator for induction of EMT in tumor metastasis. The expression or phosphorylation level of myosin II isoform regulated downstream of heterogeneous nuclear ribonucleoprotein-E1 to modulate a feature of EMT in response to TGF- $\beta .^{35}$ Therefore, ALPK1 may regulate 
both the epithelial and mesenchymal markers through activating different myosin II isoforms under external stimulators. The detailed molecular mechanisms for ALPK1promoted cell mobility via activating myosin families warrants further investigation.

Inflammatory cytokines or chemokines have biological effects on tumor development. Cytokines and chemokines are also found in the patient's serum and saliva, and may serve as diagnostic biomarkers. ${ }^{36-38}$ TNF- $\alpha$, IL-6, IL-8, and CCL5/CCR5 promote the growth, mobility, and invasion of tumor cells, and are associated with an increased rate of metastasis. ${ }^{39-42}$ In addition, high levels of TNF- $\alpha$ and IL-6 proteins were found in larger tumors (T3/T4) compared with smaller tumors $(\mathrm{T} 1 / \mathrm{T} 2){ }^{43}$ The clinicopathologic signature of ALPK1 showed that the presence of ALPK1 was strongly correlated with tumor size and involvement of lymph node metastasis (Tables 2 and 3). Previous studies suggest that ALPK1 is a transporter that can increase proinflammatory cytokine production under different stimulators, including testosterone,${ }^{11}$ monosodium urate monohydrate, ${ }^{14}$ and Shigella flexneri infection. ${ }^{17}$ Only TNF- $\alpha$ production decreased in HSC-3 cells with depleted ALPK1, but was not observed in OC3 and SAS cells. There was no difference between IL-1 $\beta$ and IL- 6 production in ALPK1-depleted cells and control cells (Supplemental Figure S2). These results indicate that ALPK1-regulated cytokine production is independent of OSCC development.

Cetuximab is used in the treatment of recurrent or metastatic OSCC. ${ }^{44,45}$ Mutation or loss of expression of EGFR status in patients may result in no response to cetuximab. ${ }^{46}$ However, tyrosine kinase inhibitors, targeting the EGFR signaling pathway, have not yet been proposed in OSCC treatment. In addition, serine/threonine kinase has been shown to promote tumor growth, survival, and drug-resistance. ${ }^{47,48}$ Currently, Food and Drug Administration-approved small-molecule kinase inhibitors have been reported to be promising targeted therapeutics. ${ }^{49}$ These findings show that ALPK1, an alphaprotein kinase, plays an important role in tumor metastasis and growth. Therefore, ALPK1 has potential as a therapeutic target in OSCCs.

Several exposure factors such as smoking, alcohol consumption, use of the areca nuts, and human papillomavirus infection are thought to result in multiple stepwise genetic alternations and ablation of molecular signaling changes that result in the formation of oral cancer. ${ }^{50}$

Additionally, these exposure factors could increase the risk of oral cancer occurrence. ${ }^{51}$ Expression of ALPK1 has also been shown to increase following monosodium urate monohydrate in gout flares. ${ }^{14}$ This result may be due to the high expression of ALPK1 in T1 stages of OSCC patients (Table 2). Thus, the interaction between genetic alternation and gene expression of ALPK1 and environmental stimulations (eg, arecoline, areca nut extract, and nicotine) need to be investigated with respect to OSCC development.

In conclusion, high ALPK1 expression was strongly related to an advanced stage of tumor size and lymph node metastasis, when evaluating clinicopathologic signatures. Further, ALPK1-depleted OSCC cells showed significantly reduced cell growth, migration, and invasion ability. Decreased mesenchymal markers were observed in knock down of ALPK1 expression on OSCC cells. However, a more detailed molecular mechanism of ALPK1-induced cellular functions needs to be investigated to evaluate the potential role in OSCC cancer progression and metastasis.

\section{Acknowledgments}

We thank Dr. Shu-Chun Lin (National Yang Ming University, Taipei, Taiwan) for providing the OC3 cells.

P.-K.C. and Y.-C.K. designed the study, integrated the results, and drafted the manuscript; C.-M.C. and Y.-C.K. analyzed data; C.-H.H., H.-T.H., T.-M.K., C.-M.C., C.-P.L., M.-H.T., and K.-T.Y. contributed reagents, materials, or analytic tools, and gave advice on experimental design; C.-H.H. and M.-H.T. helped with sample collection and obtaining the clinical information; H.-T.H. and K.-T.Y. advised on microscopic experiments; and all authors reviewed the manuscript.

\section{Supplemental Data}

Supplemental material for this article can be found at https://doi.org/10.1016/j.ajpath.2018.09.003.

\section{References}

1. Sathiyasekar AC, Chandrasekar P, Pakash A, Kumar KU, Jaishlal MS: Overview of immunology of oral squamous cell carcinoma. J Pharm Bioallied Sci 2016, 8 Suppl 1:S8-S12

2. Ermer MA, Kirsch K, Bittermann G, Fretwurst T, Vach K, Metzger MC: Recurrence rate and shift in histopathological differentiation of oral squamous cell carcinoma - a long-term retrospective study over a period of 13.5 years. J Craniomaxillofac Surg 2015, 43: 1309-1313

3. Wang B, Zhang S, Yue K, Wang XD: The recurrence and survival of oral squamous cell carcinoma: a report of 275 cases. Chin J Cancer 2013, 32:614-618

4. Allen CT, Law JH, Dunn GP, Uppaluri R: Emerging insights into head and neck cancer metastasis. Head Neck 2013, 35:1669-1678

5. Mahipal A, Kothari N, Gupta S: Epidermal growth factor receptor inhibitors: coming of age. Cancer Control 2014, 21:74-79

6. Loeffler-Ragg J, Schwentner I, Sprinzl GM, Zwierzina H: EGFR inhibition as a therapy for head and neck squamous cell carcinoma. Expert Opin Investig Drugs 2008, 17:1517-1531

7. Luo X, Crawley SW, Steimle PA, Egelhoff TT, Cote GP: Specific phosphorylation of threonine by the Dictyostelium myosin II heavy chain kinase family. J Biol Chem 2001, 276:17836-17843

8. Ryazanov AG, Pavur KS, Dorovkov MV: Alpha-kinases: a new class of protein kinases with a novel catalytic domain. Curr Biol 1999, 9: R43-R45

9. Futey LM, Medley QG, Cote GP, Egelhoff TT: Structural analysis of myosin heavy chain kinase A from Dictyostelium. Evidence for a highly divergent protein kinase domain, an amino-terminal coiled-coil domain, and a domain homologous to the beta-subunit of heterotrimeric G proteins. J Biol Chem 1995, 270:523-529 
10. Heine M, Cramm-Behrens CI, Ansari A, Chu HP, Ryazanov AG, Naim HY, Jacob R: Alpha-kinase 1, a new component in apical protein transport. J Biol Chem 2005, 280:25637-25643

11. Kuo TM, Yeh KT, Hsu HT, Chiang SL, Chang JG, Huang CM, Tu HP, Liu CS, Ko YC: ALPK1 affects testosterone mediated regulation of proinflammatory cytokines production. J Steroid Biochem Mol Biol 2015, 154:150-158

12. Ko AM, Tu HP, Liu TT, Chang JG, Yuo CY, Chiang SL, Chang SJ, Liu YF, Ko AM, Lee CH, Lee CP, Chang CM, Tsai SF, Ko YC: ALPK1 genetic regulation and risk in relation to gout. Int J Epidemiol 2013, 42:466-474

13. Wang SJ, Tu HP, Ko AM, Chiang SL, Chiou SJ, Lee SS, Tsai YS, Lee CP, Ko YC: Lymphocyte $\alpha$-kinase is a gout-susceptible gene involved in monosodium urate monohydrate-induced inflammatory responses. J Mol Med (Berl) 2011, 89:1241-1251

14. Lee CP, Chiang SL, Ko AM, Liu YF, Ma C, Lu CY, Huang CM, Chang JG, Kuo TM, Chen CL, Tsai EM, Ko YC: ALPK1 phosphorylates myosin IIA modulating TNF-alpha trafficking in gout flares. Sci Rep 2016, 6:25740

15. Yamada Y, Nishida T, Ichihara S, Kato K, Fujimaki T, Oguri M, Horibe H, Yoshida T, Watanabe S, Satoh K, Aoyagi Y, Fukuda M, Sawabe M: Identification of chromosome 3q28 and ALPK1 as susceptibility loci for chronic kidney disease in Japanese individuals by a genome-wide association study. J Med Genet 2013, 50:410-418

16. Zimmermann S, Pfannkuch L, Al-Zeer MA, Bartfeld S, Koch M, Liu J, Rechner C, Soerensen M, Sokolova O, Zamyatina A, Kosma P, Maurer AP, Glowinski F, Pleissner KP, Schmid M, Brinkmann V, Karlas A, Naumann M, Rother M, Machuy N, Meyer TF: ALPK1- and TIFA-dependent innate immune response triggered by the helicobacter pylori type IV secretion system. Cell Rep 2017, 20:2384-2395

17. Milivojevic M, Dangeard AS, Kasper CA, Tschon T, Emmenlauer M, Pique C, Schnupf P, Guignot J, Arrieumerlou C: ALPK1 controls TIFA/TRAF6-dependent innate immunity against heptose-1,7bisphosphate of gram-negative bacteria. PLoS Pathog 2017, 13: e1006224

18. Strietz J, Stepputtis SS, Preca BT, Vannier C, Kim MM, Castro DJ, Au Q, Boerries M, Busch H, Aza-Blanc P, HeynenGenel S, Bronsert P, Kuster B, Stickeler E, Brabletz T, Oshima RG, Maurer J: ERN1 and ALPK1 inhibit differentiation of bi-potential tumor-initiating cells in human breast cancer. Oncotarget 2016, 7:83278-83293

19. Liao HF, Lee HH, Chang YS, Lin CL, Liu TY, Chen YC, Yen JC, Lee YT, Lin CY, Wu SH, Ko YC, Chang JG: Down-regulated and commonly mutated ALPK1 in lung and colorectal cancers. Sci Rep 2016, 6:27350

20. Brierley JD, Gospodarowicz MK, Wittekind C (Eds): TNM Classification of Malignant Tumours. ed 8. Hoboken, NJ: John Wiley \& Sons, Inc., 2017

21. Chang PY, Kuo TM, Chen PK, Lin YZ, Hua CH, Chen YC, Ko YC: Arecoline N-oxide upregulates caspase- 8 expression in oral hyperplastic lesions of mice. J Agric Food Chem 2017, 65:10197-10205

22. Fedchenko N, Reifenrath J: Different approaches for interpretation and reporting of immunohistochemistry analysis results in the bone tissue - a review. Diagn Pathol 2014, 9:221

23. Davis FM, Stewart TA, Thompson EW, Monteith GR: Targeting EMT in cancer: opportunities for pharmacological intervention. Trends Pharmacol Sci 2014, 35:479-488

24. Scanlon CS, Van Tubergen EA, Inglehart RC, D'Silva NJ: Biomarkers of epithelial-mesenchymal transition in squamous cell carcinoma. J Dent Res 2013, 92:114-121

25. Thiery JP: Epithelial-mesenchymal transitions in tumour progression. Nat Rev Cancer 2002, 2:442-454

26. Zhou J, Tao D, Xu Q, Gao Z, Tang D: Expression of E-cadherin and vimentin in oral squamous cell carcinoma. Int J Clin Exp Pathol 2015, $8: 3150-3154$

27. Di Domenico M, Pierantoni GM, Feola A, Esposito F, Laino L, De Rosa A, Rullo R, Mazzotta M, Martano M, Sanguedolce F, Perillo L,
D’Angelo L, Papagerakis S, Tortorella S, Bufo P, Lo Muzio L, Pannone G, Santoro A: Prognostic significance of N-Cadherin expression in oral squamous cell carcinoma. Anticancer Res 2011, 31:4211-4218

28. de Araujo VC, Pinto Junior DS, de Sousa SO, Nunes FD, de Araujo NS: Vimentin in oral squamous cell carcinoma. Eur Arch Otorhinolaryngol 1993, 250:105-109

29. Chen M, Xu R: Motor coordination deficits in Alpk1 mutant mice with the inserted piggyBac transposon. BMC Neurosci 2011, 12:1

30. Vicente-Manzanares M, Ma X, Adelstein RS, Horwitz AR: Nonmuscle myosin II takes centre stage in cell adhesion and migration. Nat Rev Mol Cell Biol 2009, 10:778-790

31. Conti MA, Adelstein RS: Nonmuscle myosin II moves in new directions. J Cell Sci 2008, 121 Pt 1:11-18

32. Zhang X, Huang Z, Hu Y, Liu L: Knockdown of myosin 6 inhibits proliferation of oral squamous cell carcinoma cells. J Oral Pathol Med 2016, 45:740-745

33. Ohmura G, Tsujikawa T, Yaguchi T, Kawamura N, Mikami S, Sugiyama J, Nakamura K, Kobayashi A, Iwata T, Nakano H, Shimada T, Hisa Y, Kawakami Y: Aberrant myosin 1b expression promotes cell migration and lymph node metastasis of HNSCC. Mol Cancer Res 2015, 13:721-731

34. Ouderkirk JL, Krendel M: Non-muscle myosins in tumor progression, cancer cell invasion, and metastasis. Cytoskeleton (Hoboken) 2014, $71: 447-463$

35. Beach JR, Hussey GS, Miller TE, Chaudhury A, Patel P, Monslow J, Zheng Q, Keri RA, Reizes O, Bresnick AR, Howe PH, Egelhoff TT: Myosin II isoform switching mediates invasiveness after TGF-betainduced epithelial-mesenchymal transition. Proc Natl Acad Sci U S A 2011, 108:17991-17996

36. Sahibzada HA, Khurshid Z, Khan RS, Naseem M, Siddique KM, Mali M, Zafar MS: Salivary IL-8, IL-6 and TNF-alpha as potential diagnostic biomarkers for oral cancer. Diagnostics (Basel) 2017, 7: E21

37. Prasad G, McCullough M: Chemokines and cytokines as salivary biomarkers for the early diagnosis of oral cancer. Int J Dent 2013, 2013:813756

38. Katakura A, Kamiyama I, Takano N, Shibahara T, Muramatsu T, Ishihara K, Takagi R, Shouno T: Comparison of salivary cytokine levels in oral cancer patients and healthy subjects. Bull Tokyo Dent Coll 2007, 48:199-203

39. Tang D, Tao D, Fang Y, Deng C, Xu Q, Zhou J: TNF-alpha promotes invasion and metastasis via NF-kappa B pathway in oral squamous cell carcinoma. Med Sci Monit Basic Res 2017, 23:141-149

40. Chuang JY, Huang YL, Yen WL, Chiang IP, Tsai MH, Tang CH: Syk/JNK/AP-1 signaling pathway mediates interleukin-6-promoted cell migration in oral squamous cell carcinoma. Int J Mol Sci 2014, 15:545-559

41. Chuang JY, Yang WH, Chen HT, Huang CY, Tan TW, Lin YT, Hsu CJ, Fong YC, Tang CH: CCL5/CCR5 axis promotes the motility of human oral cancer cells. J Cell Physiol 2009, 220: $418-426$

42. Watanabe H, Iwase M, Ohashi M, Nagumo M: Role of interleukin-8 secreted from human oral squamous cell carcinoma cell lines. Oral Oncol 2002, 38:670-679

43. Nakano Y, Kobayashi W, Sugai S, Kimura H, Yagihashi S: Expression of tumor necrosis factor-alpha and interleukin-6 in oral squamous cell carcinoma. Jpn J Cancer Res 1999, 90:858-866

44. Bourhis J, Rivera F, Mesia R, Awada A, Geoffrois L, Borel C, Humblet Y, Lopez-Pousa A, Hitt R, Vega Villegas ME, Duck L, Rosine D, Amellal N, Schueler A, Harstrick A: Phase I/II study of cetuximab in combination with cisplatin or carboplatin and fluorouracil in patients with recurrent or metastatic squamous cell carcinoma of the head and neck. J Clin Oncol 2006, 24: 2866-2872

45. Vermorken JB, Trigo J, Hitt R, Koralewski P, Diaz-Rubio E, Rolland F, Knecht R, Amellal N, Schueler A, Baselga J: Open-label, 
uncontrolled, multicenter phase II study to evaluate the efficacy and toxicity of cetuximab as a single agent in patients with recurrent and/or metastatic squamous cell carcinoma of the head and neck who failed to respond to platinum-based therapy. J Clin Oncol 2007, 25: $2171-2177$

46. Kimura I, Kitahara H, Ooi K, Kato K, Noguchi N, Yoshizawa K, Nakamura H, Kawashiri S: Loss of epidermal growth factor receptor expression in oral squamous cell carcinoma is associated with invasiveness and epithelial-mesenchymal transition. Oncol Lett 2016, 11: 201-207

47. McCubrey JA, Abrams SL, Fitzgerald TL, Cocco L, Martelli AM, Montalto G, Cervello M, Scalisi A, Candido S, Libra M, Steelman LS: Roles of signaling pathways in drug resistance, cancer initiating cells and cancer progression and metastasis. Adv Biol Regul 2015, 57:75-101

48. Polivka J Jr, Janku F: Molecular targets for cancer therapy in the PI3K/AKT/mTOR pathway. Pharmacol Ther 2014, 142:164-175

49. Wu P, Nielsen TE, Clausen MH: Small-molecule kinase inhibitors: an analysis of FDA-approved drugs. Drug Discov Today 2016, 21 : $5-10$

50. Haddad RI, Shin DM: Recent advances in head and neck cancer. N Engl J Med 2008, 359:1143-1154

51. Chung CM, Lee CH, Chen MK, Lee KW, Lan CCE, Kwan AL, Tsai MH, Ko YC: Combined genetic biomarkers and betel quid chewing for identifying high-risk group for oral cancer occurrence. Cancer Prev Res (Phila) 2017, 10:355-362 